\title{
Clinical Study \\ Pelvic Exenteration: Experience from a Rural Cancer Center in Developing World
}

\author{
Sampada B. Dessai, ${ }^{1}$ Satheesan Balasubramanian, ${ }^{1}$ Vijay M. Patil, ${ }^{2}$ Santam Chakraborty, \\ Atanu Bhattacharjee, ${ }^{4}$ and Syam Vikram ${ }^{1}$ \\ ${ }^{1}$ Department of Surgical Oncology, Malabar Cancer Center, Thalassery-Koppalam-Panoor Road, Moozhikkara, Kodiyeri, \\ Thalassery, Kannur, Kerala 670103, India \\ ${ }^{2}$ Department of Clinical Hematology and Medical Oncology, Malabar Cancer Center, Thalassery-Koppalam-Panoor Road, \\ Moozhikkara, Kodiyeri, Thalassery, Kannur, Kerala 670103, India \\ ${ }^{3}$ Department of Radiation Oncology, Malabar Cancer Center, Thalassery-Koppalam-Panoor Road, Moozhikkara, Kodiyeri, \\ Thalassery, Kannur, Kerala 670103, India \\ ${ }^{4}$ Division of Clinical Research and Biostatistics, Malabar Cancer Center, Thalassery-Koppalam-Panoor Road, Moozhikkara, \\ Kodiyeri, Thalassery, Kannur, Kerala 670103, India
}

Correspondence should be addressed to Sampada B. Dessai; sampadads@gmail.com

Received 24 August 2014; Revised 29 December 2014; Accepted 5 January 2015

Academic Editor: Steven Heys

Copyright (C) 2015 Sampada B. Dessai et al. This is an open access article distributed under the Creative Commons Attribution License, which permits unrestricted use, distribution, and reproduction in any medium, provided the original work is properly cited.

Background. Pelvic exenteration (PE) is a morbid procedure. Ours is a rural based cancer center limited trained surgical oncology staff. Hence, this audit was planned to evaluate morbidity and outcomes of all patients undergoing PE at our center. Methods. This is a IRB approved retrospective audit of all patients who underwent PE at our center from January 2010 to August 2013. The toxicity grades were retrospectively assigned according to the CTCAE version 4.02 criteria. Chi-square test was done to identify factors affecting grades 3-5 morbidity. Kaplan Meier survival analysis has been used for estimation of median PFS and OS. Results. 34 patients were identified, with the median age of 52 years (28-73 years). Total, anterior, posterior, and modified posterior exenterations were performed in $4(11.8 \%), 5(14.7 \%), 14(41.2 \%)$, and $11(32.4 \%)$ patients, respectively. The median time for surgery was 5.5 hours (3-8 hours). The median blood loss was $500 \mathrm{~mL}$ (200-4000 mL). CTCAE version 4.02 grades 3-4 toxicity was seen in nine patients (25.7\%). The median estimated progression free survival was 31.76 months (25.13-38.40 months). The 2 -year overall survival was $97.14 \%$. Conclusion. PE related grades $3-5$ morbidity of $25.7 \%$ and mortality of $2.9 \%$ at our resource limited center are encouraging.

\section{Introduction}

Pelvic exenteration (PE) was first described in 1948 by Brunschwig as a form of radical surgery for recurrent carcinoma cervix [1]. As originally described, total pelvic exenteration entails en masse removal of all pelvic viscera followed by end colostomy and bilateral ureteric implantation in the colon above the stoma [1]. Several authors have described the use of this surgery in the management of recurrent and advanced neoplasms of the bladder, rectum, and other gynecological malignancies [2-5].

$\mathrm{PE}$ is an extensive surgery [6], with reported perioperative mortality rate as high as $23 \%$ [1]. However, with improvement in surgical techniques and postoperative care, a decrease in mortality has been noted in newer series [7]. Though world literature has many reports from western world about the effectiveness of pelvic exenteration, very few reports are from India $[6,8]$. This is surprising as most patients with pelvic malignancies in India present with locally advanced stages [9] and may be a related to the shortage of experienced surgical oncologists in India [10].

Outcome in surgical oncology depends on the skill of the surgeon as well as the volume of surgery performed [11]. Studies have shown that outcomes for the patients with abdominal malignancies were improved when operated upon 
in a high volume center [12]. Ours is a rural based cancer center in the public sector with a steadily increasing patient load. In addition, trained surgical oncologists were available only from 2009 onwards. Hence, this audit was planned to evaluate morbidity and outcomes of all patients undergoing pelvic exenteration at our center over three years.

\section{Material and Methods}

This is an institutional review board approved retrospective audit of all patients who underwent PE at our center from January 2010 to August 2013. Patients were identified from operation theatre registers maintained during the abovementioned time period. Data are obtained on demographic profile, primary tumor site, previous treatment, indication for PE, intraoperative and postoperative complications, adjuvant treatment, and failure. The toxicity grades were retrospectively assigned according to the Common Terminology Criteria for Adverse Events (CTCAE) version 4.02 (National Cancer Institute) criteria.

The types of pelvic exenteration were classified as below $[13,14]$ :

(1) total pelvic exenteration: hysterectomy, cystectomy, low anterior resection or APR, +/- sigmoid resection, and removal of pelvic lymph nodes;

(2) anterior pelvic exenteration with ileal conduit (Brookes technique): hysterectomy, cystectomy, and removal of pelvic lymph nodes;

(3) posterior pelvic exenteration: hysterectomy with abdominoperineal resection;

(4) modified posterior pelvic exenteration: hysterectomy with low anterior resection and coloanal anastomosis.

The postoperative care differed according to the site of tumor and according to the type of surgery. However, in general patients were kept in surgical ICU for 48 hours, they were mobilized on the second day, antibiotics were provided for 5-7 days, and deep venous thrombosis prophylaxis was provided till discharge. Oral feeds were started on second or third day depending upon the presence of stoma and/or bowel movements. Prior to this partial parenteral nutrition was provided under the guidance of the dietetics division. Stoma/ileal conduit care was provided by the specialized nursing unit along with preoperative and postoperative counselling.

During their indoor stay (calculated from day of surgery till discharge) patients were monitored as follows:

(1) hemodynamic monitoring: D0 till discharge,

(2) monitoring for arterial $\mathrm{pH}$ : D0-D1,

(3) complete hemogram, serum electrolytes, and renal function tests: performed daily from D0 till patients started full diet and required no more IV fluids.

Descriptive statistics reported include frequencies for categorical variables and median (range) for continuous variables. Chi-square test was done to identify factors associated
TABLE 1: Cross tabulation of tumor site and indication for exenteration.

\begin{tabular}{lcc}
\hline Tumor site & $\begin{array}{c}\text { Locally advanced } \\
\text { tumor beyond } \\
\text { organ of origin }\end{array}$ & $\begin{array}{c}\text { Recurrence limited } \\
\text { to pelvic organs }\end{array}$ \\
\hline $\begin{array}{l}\text { Rectum/rectosigmoid } \\
\text { Ovary }\end{array}$ & $\begin{array}{c}16 \text { patients } \\
\text { patients }\end{array}$ & $\begin{array}{c}\text { 3 patients } \\
\text { patient } \\
\text { Retroperitoneal }\end{array}$ \\
$\begin{array}{l}\text { sarcoma } \\
\text { Bladder }\end{array}$ & 00 & 1 patient \\
\hline
\end{tabular}

with grades 3-5 morbidity. Kaplan Meier survival analysis was used for estimation of median progression free survival (PFS) and overall survival (OS). PFS was calculated from the date of surgery till date of progression or death, whichever was earlier. Patients were censored at the last date of followup if still not progressed. OS was calculated from the date of surgery till date of death, with patients being censored at the last date of follow-up if still alive. SPSS version 16 was used for analysis.

\section{Results}

3.1. Demographic Pattern. Thirty-four patients were identified subjected to the above-mentioned criteria. Figure 1 provides the consort diagram. The median age was 52 years (28-73 years). There were 28 females and 6 male patients. All patients had ECOG PS 0-1. Nine patients had hypertension and six patients had diabetes mellitus, all of which were medically controlled. In twenty-nine patients the exenteration was done as a part of the primary treatment, while in five patients it was done for recurrent disease. In the 5 patients with recurrence, the median disease free interval was 14 months (range 9-48 months) prior to PE.

3.2. Tumor Details and Indication of Exenteration. The details of primary tumor and indication of exenteration are shown in Table 1. The primary site of tumor was in rectum or sigmoid in 19 patients, in ovary or endometrium in 11 patients, in bladder in 3 patients, and in retroperitoneum in one patient. The indications for performing a PE were locally advanced tumor extending beyond the organ of origin in 29 patients and recurrence limited to pelvis in 5 patients. In 15 patients with rectal cancer preoperative chemoradiation was given. In nine patients with upfront ovarian cancer neoadjuvant chemotherapy (NACT) was given using three weekly paclitaxel and carboplatin.

3.3. Surgical Details. Total, anterior, posterior, and modified posterior exenterations were performed in $4(11.8 \%)$, $5(14.7 \%), 14(41.2 \%)$, and $11(32.4 \%)$ patients, respectively. Intraoperatively in 7 patients bladder was involved, in 4 patients disease was extending up to the pelvic side walls, and in 2 patients sacrum was involved. All patients had R0 


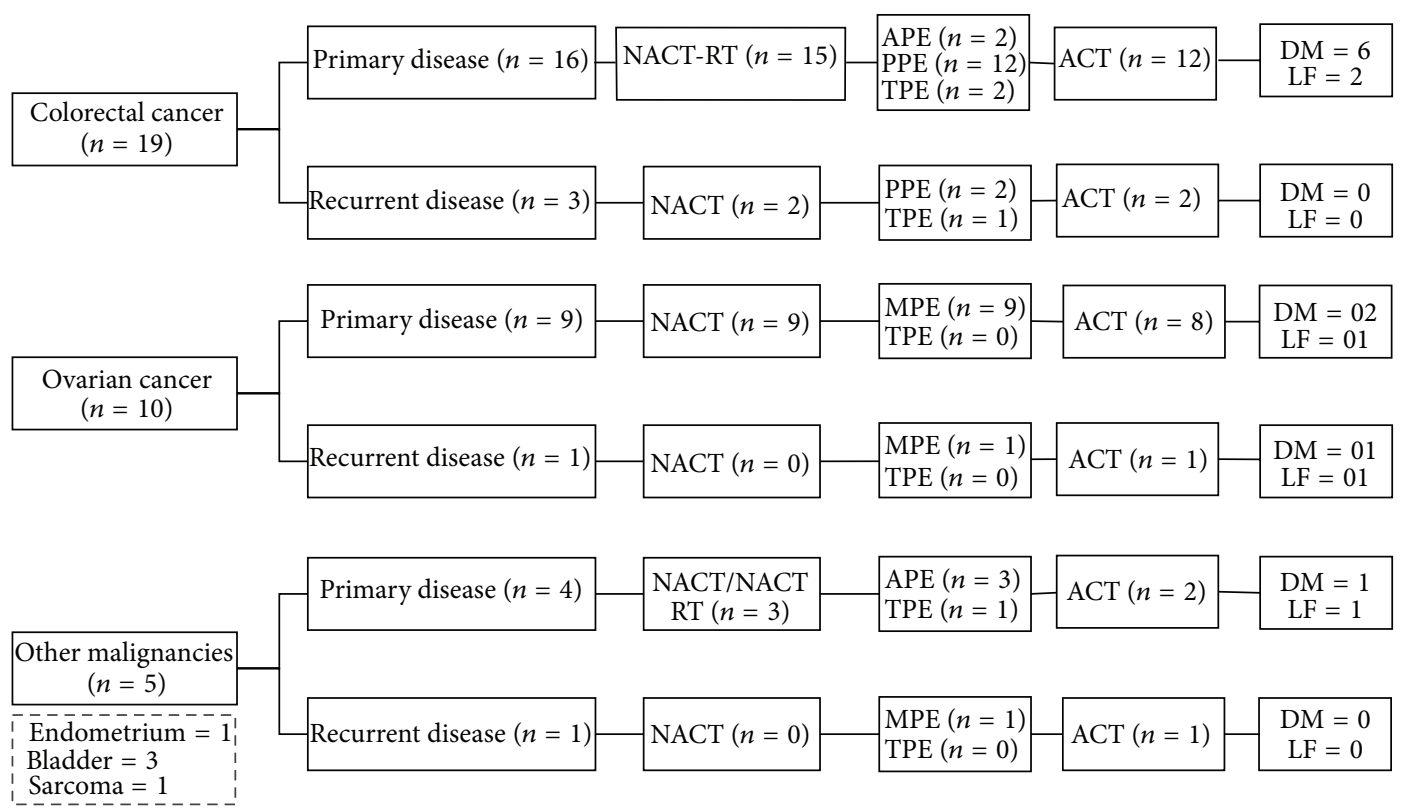

FIGURE 1: Consort diagram of patients. NACT: neoadjuvant chemotherapy, NACT-RT: neoadjuvant chemoradiation, APE: anterior pelvic exenteration, PPE: posterior pelvic exenteration, MPE: modified pelvic exenteration, TPE: total pelvic exenteration, ACT: adjuvant chemotherapy, LF: local failure, and DF: distant failure.

TABLE 2: Details of lymph node dissection performed.

\begin{tabular}{ll}
\hline Variable & Value \\
\hline Lymph node dissection done & 33 patients \\
Type of LN dissection & \\
Pelvic + paraaortic & 11 patients \\
Pelvic & 21 patients \\
Pericolic & 01 patient \\
Median number of LN retrieved & 12 nodes (1-39 nodes) \\
\hline
\end{tabular}

resection. Sacral resection was done in 2 patients and accompanying lymph node dissection was done in 33 patients. In the patient with retroperitoneal sarcoma lymph node dissection was not done. The details of lymph node dissection done are given in Table 2. The median time for surgery was 5.5 hours (3-8 hours). The median blood loss was $500 \mathrm{~mL}$ (200$4000 \mathrm{~mL})$.

3.4. Complications. The median interval of indoor admission was 11 days (6-32 days). CTCAE version 4.02 grades 34 toxicity was seen in nine patients $(25.7 \%)$. One patient succumbed to postoperative sepsis on the 12th postoperative day. Postoperative ICU admissions were required in all patients. The details of other toxicity are shown in Table 3.

There was no difference in grades 3-5 morbidity according to age and type of exenteration or according to the disease status (primary or recurrent). Though the rate of grades 35 morbidity was $36.66 \%$ in patients with age 60 years and above, as opposed to $20.8 \%$ in patients below the age of 60 years, it was not statistically significant. $(P=0.329)$. The factor which affected grades 3-5 morbidity was site of primary. In patients with nonrectal primary the incidence of grades $3-5$ morbidity was $11.1 \%$ versus $41.2 \%$ in patients having a rectal primary $(P=0.042)$.

Details of other toxicities are shown in Table 3. There was no difference in grades 3-5 morbidity according to age and type of exenteration or according to the disease status (primary or recurrent). Though the rate of grades 35 morbidity was $36.66 \%$ in patients with age 60 years and above, as opposed to $20.8 \%$ in patients below the age of 60 years, it was not statistically significant $(P=0.329)$. The factor which affected grades 3-5 morbidity was site of primary. In patients with nonrectal primary the incidence of grades $3-5$ morbidity was $11.1 \%$ as compared to $41.2 \%$ in patients having a rectal primary $(P=0.042)$.

3.5. Adjuvant Treatment. Adjuvant treatment was received in 28 patients. Adjuvant chemotherapy was delivered in 26 patients while adjuvant radiation was given in 2 patients, both of whom had rectal cancers.

Among ovarian cancer patients, as noted above, 9 patients had received NACT expect one rest all 8 completed adjuvant chemotherapy. They all received total 6 cycles of paclitaxel and carboplatin. One patient of ovarian cancer developed a myocardial infarction postoperatively with a decline in ejection fraction which precluded further adjuvant treatment. One patient with ovarian cancer who underwent exenteration for recurrent disease received 6 cycles of adjuvant gemcitabine and carboplatin regimen.

In 20 patients with colorectal cancer, 17 patients received capecitabine oxaliplatin regimen for 8 cycles, while 3 patients declined further adjuvant chemotherapy. All these 3 patients had rectal cancers, one of whom had received upfront 
TABLE 3: Acute toxicity within 30 days of surgery.

\begin{tabular}{|c|c|c|c|c|c|}
\hline & Grade 1 & Grade 2 & Grade 3 & Grade 4 & Grade 5 \\
\hline $\begin{array}{l}\text { Intraoperative } \\
\text { bowel/ureter/venous injury }\end{array}$ & 0 & 0 & 0 & 0 & 0 \\
\hline Wound infection & 1 & 4 & 2 & 0 & 0 \\
\hline Colonic fistula & 0 & 0 & 0 & 0 & 0 \\
\hline Small intestinal obstruction & 0 & 0 & 0 & 0 & 0 \\
\hline Ureteric anastomotic leak & 0 & 0 & 0 & 0 & 0 \\
\hline Postoperative hemorrhage & 0 & 0 & 1 & 0 & 0 \\
\hline Ventricular arrhythmia & 1 & 0 & 0 & 0 & 0 \\
\hline Skin ulceration ${ }^{*}$ & 0 & 0 & 0 & 0 & 0 \\
\hline Rise in serum creatinine & 0 & 2 & 0 & 0 & 0 \\
\hline Metabolic acidosis & 1 & 0 & 1 & 0 & 0 \\
\hline Sepsis & 2 & 0 & 0 & 0 & 1 \\
\hline Hyponatremia & 16 & NA & 6 & 0 & 0 \\
\hline Hypernatremia & 3 & 1 & 0 & 0 & 0 \\
\hline Hypokalemia & 13 & 7 & 1 & 0 & 0 \\
\hline Hyperkalemia & 0 & 1 & 1 & 0 & 0 \\
\hline Hypomagnesemia & 0 & 0 & 0 & 0 & 0 \\
\hline Anemia & 16 & 10 & 4 & 0 & 0 \\
\hline Thromboembolic event ${ }^{* *}$ & 0 & 1 & 0 & 0 & 0 \\
\hline Myocardial infarction & 0 & 0 & 0 & 1 & 0 \\
\hline
\end{tabular}

Numbers depicted are numbers of patients. ${ }^{*}$ Ulceration of skin due to pressure ulcer. ${ }^{* *}$ The event was deep venous thrombosis.

TABLE 4: Table depicting outcomes (overall survival and progression free survival) in major sites.

\begin{tabular}{lccc}
\hline & $N$ & $\begin{array}{c}\text { Two-year PFS } \\
(95 \% \text { CI })\end{array}$ & Two-year OS \\
\hline Colorectal cancer & 20 & $\begin{array}{c}62.5 \% \\
(39-100 \%)\end{array}$ & $\begin{array}{c}94.7 \% \\
(85-100 \%)\end{array}$ \\
Ovarian cancer & 10 & $\begin{array}{c}37.5 \% \\
(08-100 \%)\end{array}$ & $100 \%$ \\
\hline
\end{tabular}

chemoradiation. Adjuvant pelvic radiation was delivered to the other 2 patients to a total dose of $50.4 \mathrm{~Gy}$ in 28 fractions.

3.6. Failure Pattern. At a mean follow-up of 18 months, 10 patients had progressed. The predominant site of failure was systemic in 8 patients. Three patients had local failure only, while 2 patients had local with systemic failure.

3.7. Survival. The median estimated progression free survival was 31.76 months (25.13-38.40 months). The median overall survival was not reached. Only one patient had died. The 2-year overall survival was $97.14 \%$. Outcomes according to different sites of tumor are depicted in Table 4.

\section{Discussion}

Pelvic exenteration is an important procedure indicated in various gynecological, gastrointestinal, and other pelvic tumors $[3,5,15]$. Traditionally this procedure is associated with high mortality and morbidity. Mortality rates as high as $23 \%$ were described in mid-twentieth century with a mean time of mortality of 8 days [1]. Subsequently, however, refinements in surgical techniques with better supportive care facilities have decreased the mortality associated with this procedure to below $5-10 \%[7,16-18]$. However, still considerable proportion of patients (23-44\%) have severe morbidity associated with this procedure [7, 16-18]. In our series, the rate of grades 3-5 toxicity was $25.7 \%$ and postoperative mortality within 30 days was $2.9 \%$. These figures are in concurrence with major series reported in the twenty first century [16-22].

Ours is a rural based cancer center with limited manpower. From 2010 to 2013, only 2 trained surgical oncologists were working at this center catering to more than 2000 patients each per year. In addition, there was only one full time anesthetist working during this period at the center. The institute was devoid of a critical care specialist until recently which may be the underlying reason behind majority of grades 3-5 adverse events being biochemical and hematological disturbances. In spite of these shortcomings, the overall results of morbidity and mortality are encouraging.

The institute started doing these surgeries from 2010. It is known that, in oncological surgeries, high volumes and standardization of techniques lead to better surgical outcomes $[11,12]$. Selected experiences reported on pelvic exenteration surgeries from other centers show that on average 2-3 pelvic exenterative surgeries are done per year [7, 19-22]. At our center on average we do nearly 10 PE per year. Moreover, there has been a steady increase in these surgeries from 3 
exenterations been done in 2010 to 15 done till August in the year 2013.

The reasons for this low mortality and morbidity are just not limited to the high volume and surgical experience at our centre. The selection criteria for such surgeries need to be stringent. In our series, all patients had ECOG PS0-1. None of these patients had uncontrolled comorbidities. The median age was near 50 years. This means that fairly young patients with good performance status were selected. In addition, most of the surgeries were performed in the primary setting which is known to be associated with reduced morbidity as compared to surgery in the setting of recurrent disease $[19,23,24]$.

Posterior exenteration or modified posterior exenteration was done in majority of patients in this series, as majority of the patients had colorectal primaries. Modified posterior exenteration procedure has same amount of morbidity as a cytoreductive surgery at least in ovarian cancers [25]. All patients had received prophylactic antibiotics, DVT prophylaxis, and intensive nursing care in the postoperative period. All these factors taken together may have contributed to the low mortality and morbidity in this series [7]. The single mortality seen in this series was that of a young patient who had undergone total pelvic exenteration for a recurrence of rectal cancer. She had sepsis with blood culture positive for colistin resistant Acinetobacter baumannii and succumbed to the infection on the 12th postoperative day.

Our rate of surgical R0 resection compares favourably with other series reporting an $\mathrm{R} 0$ resection rate of $46.3 \%$ $68.4 \%$ which have been reported [20, 26-28]. Thus, decision regarding operability in such patients should be based on a combination of clinical and radiological findings. However, $\mathrm{R} 1$ and R2 pelvic exenteration resections are known to be associated with poor prognosis and should be avoided [20, 26-28].

The predominantly distant failure pattern seen in the present series is intriguing, as majority of the patients had rectal cancers and received systemic chemotherapy. However, such a predominant systemic mode of failure has also been reported in the literature in both rectal and gynecological primaries treated with pelvic exenteration $[29,30]$.

The limitations of the present study were a mixed patient population, modest sample size, limited follow-up, and a retrospective design. However, these surgeries were performed in a two-year time period without significant heterogeneity in treatment policies. In order to minimize bias we have reported outcomes of all patients undergoing PE during the time period. However, a median PFS of nearly 32 months is a promising finding especially in light of the resource limitations. The two-year overall survival achieved is $97.14 \%$ which is in line with outcomes reported for patients undergoing primary $\mathrm{PE}$ in modern series $[6,20,27,31,32]$.

\section{Conclusion}

PE related grades 3-5 morbidity of $25.7 \%$ and mortality of $2.9 \%$ at our resource limited center are encouraging. Further improvements in outcomes are expected with increased availability of critical care expertise. These results should encourage the uptake of this treatment modality in other centres in resource limited nations with a higher burden of advanced pelvic malignancies.

\section{Conflict of Interests}

The authors declare no conflict of interests.

\section{References}

[1] A. Brunschwig, "Complete excision of pelvic viscera for advanced carcinoma: a one-stage abdominoperineal operation with end colostomy and bilateral ureteral implantation into the colon above the colostomy," Cancer, vol. 1, no. 2, pp. 177-183, 1948.

[2] H. Ike, H. Shimada, S. Yamaguchi, Y. Ichikawa, S. Fujii, and S. Ohki, "Outcome of total pelvic exenteration for primary rectal cancer," Diseases of the Colon and Rectum, vol. 46, no. 4, pp. 474480, 2003.

[3] T. X. Yang, D. L. Morris, and T. C. Chua, "Pelvic exenteration for rectal cancer: a systematic review," Diseases of the Colon and Rectum, vol. 56, no. 4, pp. 519-531, 2013.

[4] M. A. Terán-Porcayo, I. Zeichner-Gancz, R. A. C. Gomez del-Castillo, A. Beltrán-Ortega, and G. Solorza-Luna, "Pelvic exenteration for recurrent or persistent cervical cancer," Medical Oncology, vol. 23, no. 2, pp. 219-223, 2006.

[5] E. J. Diver, J. A. Rauh-Hain, and M. G. del Carmen, "Total pelvic exenteration for gynecologic malignancies," International Journal of Surgical Oncology, vol. 2012, Article ID 693535, 9 pages, 2012.

[6] R. Ramamurthy and A. Duraipandian, "Morbidity and outcome of pelvic exenteration in locally advanced pelvic malignancies," Indian Journal of Surgical Oncology, vol. 3, no. 3, pp. 231-235, 2012.

[7] J. M. Goldberg, M. S. Piver, R. E. Hempling, C. Aiduk, L. Blumenson, and F. O. Recio, "Improvements in pelvic exenteration: factors responsible for reducing morbidity and mortality," Annals of Surgical Oncology, vol. 5, no. 5, pp. 399-406, 1998.

[8] P. Durgatosh, Z. Shuaib, M. Vikas, and K. Ravi, "Pelvic exenteration: a perspective from a regional cancer center in India," Indian Journal of Cancer, vol. 41, no. 3, pp. 109-114, 2004.

[9] M. Sarkar, H. Konar, and D. Raut, "Clinico-pathological features of gynecological malignancies in a tertiary care hospital in Eastern India: importance of strengthening primary health care in prevention and early detection," Asian Pacific Journal of Cancer Prevention, vol. 14, no. 6, pp. 3541-3547, 2013.

[10] R. A. Badwe and C. S. Pramesh, "Surgical oncology-at the crossroads," Indian Journal of Surgery, vol. 71, no. 6, pp. 290291, 2009.

[11] M. Gonen, D. Rubin, M. Radzyner, and M. F. Brennan, "Long-term survival is superior after resection for cancer in high-volume centers," Annals of Surgery, vol. 242, no. 4, pp. 540-454, 2005, http://www.ncbi.nlm.nih.gov/pmc/articles/ PMC1402350.

[12] S. V. Shrikhande, S. G. Barreto, B. A. Somashekar et al., "Evolution of pancreatoduodenectomy in a tertiary cancer center in India: improved results from service reconfiguration," Pancreatology, vol. 13, no. 1, pp. 63-71, 2013. 
[13] M. A. Rodriguez-Bigas and N. J. Petrelli, "Pelvic exenteration and its modifications," American Journal of Surgery, vol. 171, no. 2, pp. 293-301, 1996.

[14] A. B. McEwan and P. Clark, "The stoma of the ileal conduit," British Journal of Urology, vol. 45, no. 6, pp. 600-605, 1973.

[15] M. P. Kuhrt, R. J. Chokshi, D. Arrese, and E. W. Martin Jr., "Retrospective review of pelvic malignancies undergoing total pelvic exenteration," World Journal of Surgical Oncology, vol. 10, article 110, 2012.

[16] S. Marnitz, C. Köhler, M. Müller, K. Behrens, K. Hasenbein, and A. Schneider, "Indications for primary and secondary exenterations in patients with cervical cancer," Gynecologic Oncology, vol. 103, no. 3, pp. 1023-1030, 2006.

[17] S. Sharma, K. Odunsi, D. Driscoll, and S. Lele, "Pelvic exenterations for gynecological malignancies: twenty-year experience at Roswell Park Cancer Institute," International Journal of Gynecological Cancer, vol. 15, no. 3, pp. 475-482, 2005.

[18] A. Maggioni, G. Roviglione, F. Landoni et al., "Pelvic exenteration: ten-year experience at the European Institute of Oncology in Milan," Gynecologic Oncology, vol. 114, no. 1, pp. 64-68, 2009.

[19] T. S. Domes, P. H. D. Colquhoun, B. Taylor, J. I. Izawa, A. A. House, and P. P. W. Luke, "Total pelvic exenteration for rectal cancer: outcomes and prognostic factors," Canadian Journal of Surgery, vol. 54, no. 6, pp. 387-393, 2011.

[20] C. Fotopoulou, U. Neumann, R. Kraetschell et al., "Longterm clinical outcome of pelvic exenteration in patients with advanced gynecological malignancies," Journal of Surgical Oncology, vol. 101, no. 6, pp. 507-512, 2010.

[21] M. Kaur, S. Joniau, A. D’Hoore et al., "Pelvic exenterations for gynecological malignancies: a study of 36 cases," International Journal of Gynecological Cancer, vol. 22, no. 5, pp. 889-896, 2012.

[22] T. Benn, R. A. Brooks, Q. Zhang et al., "Pelvic exenteration in gynecologic oncology: a single institution study over 20 years," Gynecologic Oncology, vol. 122, no. 1, pp. 14-18, 2011.

[23] H. E. Averette, M. Lichtinger, B. U. Sevin, and R. E. Girtanner, "Pelvic exenteration: a 15 -year experience in a general metropolitan hospital," The American Journal of Obstetrics and Gynecology, vol. 150, no. 2, pp. 179-184, 1984.

[24] J. W. Orr Jr., H. M. Shingleton, K. D. Hatch, P. T. Taylor, E. E. Partridge, and S. J. Soong, "Gastrointestinal complications associated with pelvic exenteration," The American Journal of Obstetrics and Gynecology, vol. 145, no. 3, pp. 325-332, 1983.

[25] A. Revaux, R. Rouzier, M. Ballester, F. Selle, E. Daraï, and E. Chéreau, "Comparison of morbidity and survival between primary and interval cytoreductive surgery in patients after modified posterior pelvic exenteration for advanced ovarian cancer," International Journal of Gynecological Cancer, vol. 22, no. 8, pp. 1349-1354, 2012.

[26] H. Tixier, J. Fraisse, B. Chauffert et al., "Evaluation of pelvic posterior exenteration in the management of advanced-stage ovarian cancer," Archives of Gynecology and Obstetrics, vol. 281, no. 3, pp. 505-510, 2010.

[27] T. Ueda, F. Koyama, T. Nakagawa et al., "Clinical outcomes of pelvic exenteration for locally advanced primary or recurrent non-colorectal pelvic malignancies," Gan to Kagaku Ryoho. Cancer \& Chemotherapy, vol. 40, no. 12, pp. 2433-2436, 2013.

[28] A.-M. Schmidt, P. Imesch, D. Fink, and H. Egger, "Indications and long-term clinical outcomes in 282 patients with pelvic exenteration for advanced or recurrent cervical cancer," Gynecologic Oncology, vol. 125, no. 3, pp. 604-609, 2012.
[29] M. Jurado, J. L. Alcázar, and R. Martinez-Monge, "Resectability rates of previously irradiated recurrent cervical cancer (PIRCC) treated with pelvic exenteration: is still the clinical involvement of the pelvis wall a real contraindication? A twenty-year experience," Gynecologic Oncology, vol. 116, no. 1, pp. 38-43, 2010.

[30] P. Luna-Perez, S. Delgado, S. Labastida, N. Ortiz, D. Rodriguez, and Herrera L., "Patterns of recurrence following pelvic exenteration and external radiotherapy for locally advanced primary rectal adenocarcinoma," Annals of Surgical Oncology, vol. 3, no. 6, pp. 526-533, 1996.

[31] A. Bhangu, S. Mohammed Ali, G. Brown, R. J. Nicholls, and P. Tekkis, "Indications and outcome of pelvic exenteration for locally advanced primary and recurrent rectal cancer," Annals of Surgery, vol. 259, no. 2, pp. 315-322, 2014.

[32] F. T. J. Ferenschild, M. Vermaas, C. Verhoef et al., "Total pelvic exenteration for primary and recurrent malignancies," World Journal of Surgery, vol. 33, no. 7, pp. 1502-1508, 2009. 


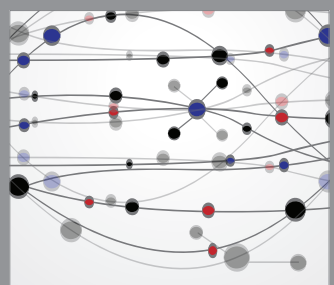

The Scientific World Journal
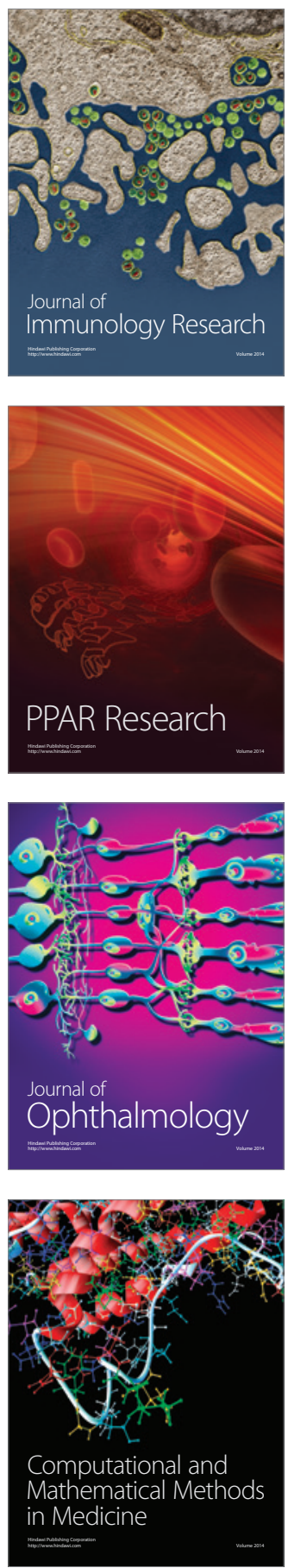

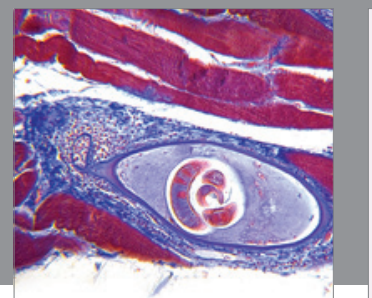

Gastroenterology

Research and Practice
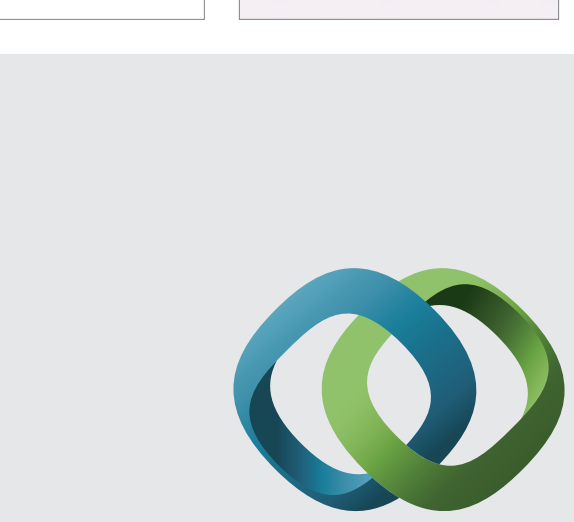

\section{Hindawi}

Submit your manuscripts at

http://www.hindawi.com
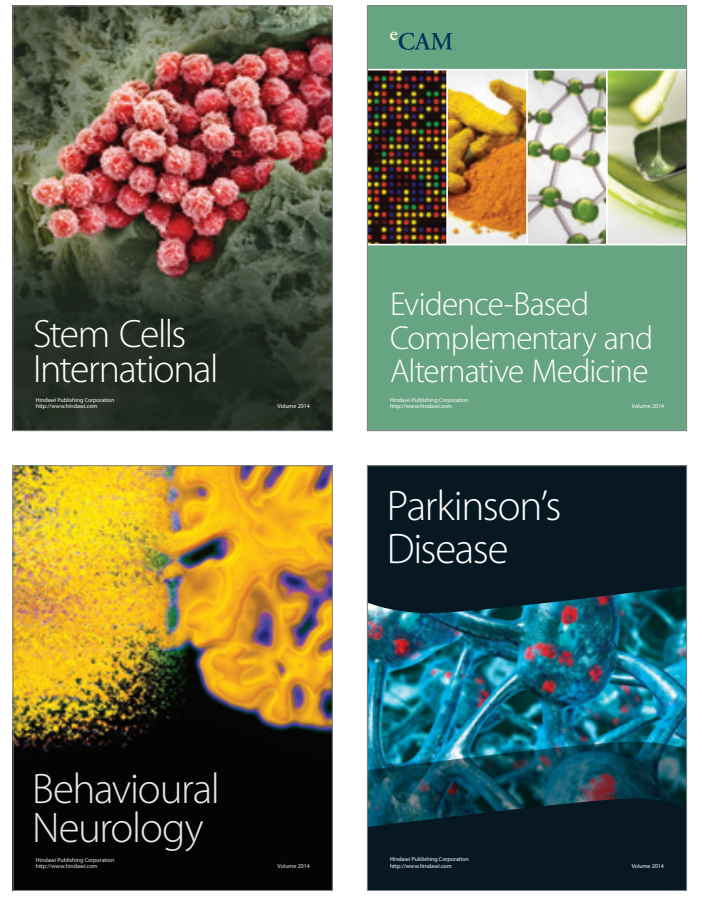
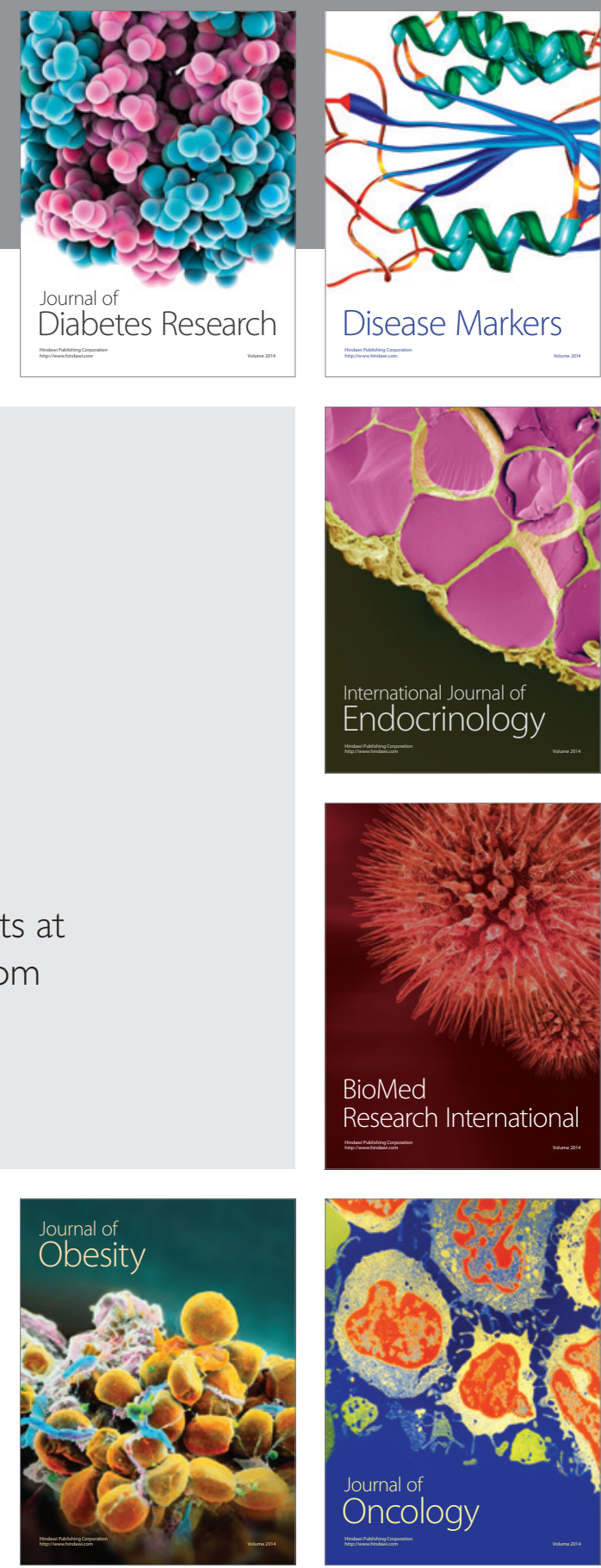

Disease Markers
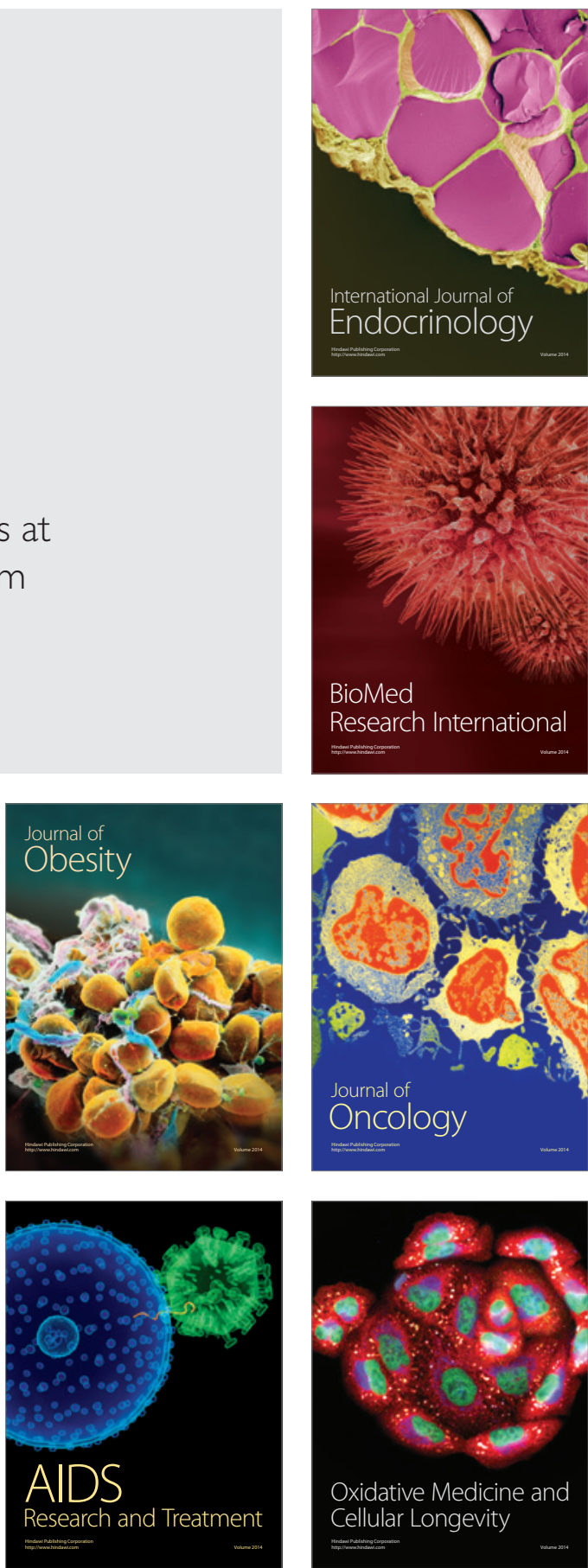